University of Vermont

UVM ScholarWorks

$1-1-2014$

\title{
A framework for evaluating the influence of climate, dispersal limitation, and biotic interactions using fossil pollen associations across the late Quaternary
}

\author{
Jessica L. Blois \\ UC Merced \\ Nicholas J. Gotelli \\ University of Vermont \\ Anna K. Behrensmeyer \\ Smithsonian National Museum of Natural History \\ J. Tyler Faith \\ The University of Queensland \\ S. Kathleen Lyons \\ Smithsonian National Museum of Natural History
}

See next page for additional authors

Follow this and additional works at: https://scholarworks.uvm.edu/casfac

Part of the Climate Commons

\section{Recommended Citation}

Blois JL, Gotelli NJ, Behrensmeyer AK, Faith JT, Lyons SK, Williams JW, Amatangelo KL, Bercovici A, Du A, Eronen JT, Graves GR. A framework for evaluating the influence of climate, dispersal limitation, and biotic interactions using fossil pollen associations across the late Quaternary. Ecography. 2014 Nov;37(11):1095-108.

This Article is brought to you for free and open access by the College of Arts and Sciences at UVM ScholarWorks. It has been accepted for inclusion in College of Arts and Sciences Faculty Publications by an authorized administrator of UVM ScholarWorks. For more information, please contact scholarworks@uvm.edu. 


\section{Authors}

Jessica L. Blois, Nicholas J. Gotelli, Anna K. Behrensmeyer, J. Tyler Faith, S. Kathleen Lyons, John W. Williams, Kathryn L. Amatangelo, Antoine Bercovici, Andrew Du, Jussi T. Eronen, Gary R. Graves, Nathan Jud, Conrad Labandeira, Cindy V. Looy, Brian Mcgill, David Patterson, Richard Potts, Brett Riddle, Rebecca Terry, Anikó Tóth, Amelia Villaseñor, and Scott Wing 


\title{
A framework for evaluating the influence of climate, dispersal limitation, and biotic interactions using fossil pollen associations across the late Quaternary
}

\author{
Jessica L. Blois, Nicholas J. Gotelli, Anna K. Behrensmeyer, J. Tyler Faith, S. Kathleen Lyons, \\ John W. Williams, Kathryn L. Amatangelo, Antoine Bercovici, Andrew Du, Jussi T. Eronen, \\ Gary R. Graves, Nathan Jud, Conrad Labandeira, Cindy V. Looy, Brian McGill, David Patterson, \\ Richard Potts, Brett Riddle, Rebecca Terry, Anikó Tóth, Amelia Villaseñor and Scott Wing
}

J. L. Blois (jblois@ucmerced.edu), School of Natural Sciences, Univ. of California, Merced, 5200 North Lake Road, Merced, CA 95343, USA. - N. J. Gotelli, Dept of Biology, Univ. of Vermont, Burlington, VT 05405, USA. - A. K. Behrensmeyer, S. K. Lyons, C. Labandeira, A. Toth and S. Wing, Dept of Paleobiology, and ETE Program, National Museum of Natural History, Smithsonian Inst., Washington, DC, USA. CL also at: Dept of Entomology, Univ. of Maryland College Park, College Park, MD, USA. - J. T. Faith, School of Social Science, The Univ. of Queensland, Brisbane, QLD 4072, Australia. - J. W. Williams, Dept of Geography and Center for Climatic Research, Univ. of WisconsinMadison, 550 North Park St, Madison, WI 53706, USA. - K. L. Amatangelo, Environmental Science and Biology, The College at BrockportSUNY, Brockport, NY, USA. - A. Bercovici, Dept of Geology, Lund Univ., Lund, Sweden. - A. Du, D. Patterson and A. Villaseñor, Hominid Paleobiology Doctoral Program, Center for the Advanced Study of Hominid Paleobiology, Dept of Anthropology, George Washington Univ., Washington, DC, USA. - J. T. Eronen, Dept of Geosciences and Geography, Univ. of Helsinki, Helsinki, Finland. - G. R. Graves, Dept of Vertebrate Zoology, National Museum of Natural History, Smithsonian Inst., Washington, DC, USA, and Center for Macroecology, Evolution and Climate, Univ. of Copenhagen, Copenhagen, Denmark. - N. Jud, Biological Sciences Graduate Program, Univ. of Maryland, College Park, MD, USA. - C. V. Looy, Dept of Integrative Biology, and Museum of Paleontology, Univ. of California Berkeley, Berkeley, CA, USA. - B. McGill, School Biology and Ecology and Sustainability Solutions Initiative, Univ. of Maine, Orono, ME, USA. - R. Potts, Dept of Anthropology, Human Origins Program, National Museum of Natural History, Smithsonian Inst., Washington, DC, USA. - B. Riddle, School of Life Sciences, Univ. of Nevada-Las Vegas, Las Vegas, NV, USA. - R. Terry, Dept of Integrative Biology, Oregon State Univ., Corvallis, OR, USA.

\begin{abstract}
Environmental conditions, dispersal lags, and interactions among species are major factors structuring communities through time and across space. Ecologists have emphasized the importance of biotic interactions in determining local patterns of species association. In contrast, abiotic limits, dispersal limitation, and historical factors have commonly been invoked to explain community structure patterns at larger spatiotemporal scales, such as the appearance of late Pleistocene no-analog communities or latitudinal gradients of species richness in both modern and fossil assemblages. Quantifying the relative influence of these processes on species co-occurrence patterns is not straightforward. We provide a framework for assessing causes of species associations by combining a null-model analysis of co-occurrence with additional analyses of climatic differences and spatial pattern for pairs of pollen taxa that are significantly associated across geographic space.

We tested this framework with data on associations among 106 fossil pollen taxa and paleoclimate simulations from eastern North America across the late Quaternary. The number and proportion of significantly associated taxon pairs increased over time, but only 449 of 56194 taxon pairs were significantly different from random. Within this significant subset of pollen taxa, biotic interactions were rarely the exclusive cause of associations. Instead, climatic or spatial differences among sites were most frequently associated with significant patterns of taxon association. Most taxon pairs that exhibited co-occurrence patterns indicative of biotic interactions at one time did not exhibit significant associations at other times. Evidence for environmental filtering and dispersal limitation was weakest for aggregated pairs between 16 and $11 \mathrm{kyr}$ BP, suggesting enhanced importance of positive species interactions during this interval. The framework can thus be used to identify species associations that may reflect biotic interactions because these associations are not tied to environmental or spatial differences. Furthermore, temporally repeated analyses of spatial associations can reveal whether such associations persist through time.
\end{abstract}

In biogeography and community ecology, there is a long tradition of using null model analysis to detect association patterns among species (Harvey et al. 1983, Wiens 1989, Gotelli and Graves 1996). Although these analyses are effective at revealing non-random species associations, relatively little attention has been devoted to understanding the underlying causes of non-random patterns (Collins et al. 2011, Gotelli and Ulrich 2012). It is clear that environmental factors (e.g. habitat differences and climate gradients) have been and continue to be major factors structuring many 
aspects of communities through time and across space (Ackerly 2003, Blois et al. 2013a, Dalsgaard et al. 2013). However, interactions among species also have played a major role in structuring community composition and functioning (Jablonski 2008, Blois et al. 2013c, Wisz et al. 2013). Given recent interest in understanding how climate change may lead to new biotic interactions and unexpected ecological dynamics (Zarnetske et al. 2012, Blois et al. 2013c), there is a critical need to disentangle the joint effects of abiotic and biotic factors on community dynamics.

Previous work on assemblage structure has quantified community pattern as a single index - such as the number of species or the number of checkerboard pairs - that is then subject to null model analysis. Even a moderatelysized assemblage (e.g. a dataset with multiple sites and multiple species at each site) contains many potential species pairs, however, each of which may exhibit positive, negative, or random associations. In many cases, single metrics that summarize an entire assemblage can be deceptive (Ulrich and Gotelli 2012), and it is more instructive to analyze individual pairs of species (Sfenthourakis et al. 2006). Gotelli and Ulrich (2010) use an empirical Bayes approach (Efron 2005) to control for the potentially large number of false positives that can emerge with the analysis of many species pairs. This kind of analysis allows for a determination of the relative frequency of positively, negatively, and randomly associated species pairs. However, non-random species associations are not necessarily caused by species interactions. Thus, a central dilemma is how to distinguish non-random species associations produced by actual species interactions from those produced by environmental filtering or dispersal limitations. All three processes can operate singly or in concert to generate both positive and negative species associations.

Ecologists working with modern faunas often explicitly or implicitly limit comparisons to a set of environmentally similar and spatially adjacent sites for which dispersal limitation is unlikely to be important (Phillips et al. 2003, Zhang et al. 2011). In such systems, it is reasonable to attribute non-random species associations to species interactions. The effects of species interactions certainly can be scaled up to larger spatial and temporal domains (Jablonski 2008, Gilman et al. 2010, Baiser et al. 2012, Blois et al. 2013c), and MacArthur (1972) argued explicitly for this scaling in his final book, Geographical ecology. However, the effects of environment, dispersal, and history become progressively more important at larger spatial and temporal scales, and it is difficult to untangle them from the effects of species interactions (Ricklefs 2004).

This dilemma is illustrated clearly in fossil records. These records usually encompass timescales at which environment, dispersal, and biotic interactions are all potentially important controls on species distributions and the associations among species, yet usually their effects cannot be directly observed. Often we have information only about species occurrences across space and through time, and perhaps information about past environments. Only rarely can we infer actual biotic interactions in fossil systems (Wilf et al. 2001, Kowalewski 2002, Currano et al. 2010, Peñalver et al. 2012, Blois et al. 2013c), making it difficult to confidently attribute the causes of past species associations to the influence of environmental similarity, interactions with other species, or other factors.

Here, we provide a framework for inferring the importance of biotic interactions, dispersal limitation, and abiotic effects on positive and negative species associations. This framework can be applied to species associations measured at any spatial or temporal scale, but we illustrate it in an analysis of eastern North American plant assemblages based on fossil pollen data from the past 21000 yr. Previous work on both individual species and communities has demonstrated that changes in fossil pollen assemblages across space and time are tightly linked with climate, particularly in the latest Pleistocene and early Holocene (Grimm et al. 1993, Williams et al. 2002, Shuman et al. 2004, Yu 2007, Blois et al. 2013a). Indeed, the tight linkages between vegetation and climate make fossil pollen data an excellent proxy for reconstructing past climates (Viau et al. 2006, Bartlein et al. 2011). Additionally, the recognition of individualistic species responses to deglaciation, the resulting formation of noanalog communities during the Pleistocene-Holocene transition, and the attribution of these communities to no-analog climates (Williams et al. 2004) suggest that species interactions should not be dominant drivers of community patterns, especially during the height of the no-analog period from 17-11 kyr BP. Other evidence suggests that not all changes in vegetation can be attributed exclusively to climate. For example, at several sites in the Great Lakes region, the loss of megaherbivores and their associated species interactions at the end of the Pleistocene may have contributed to the formation of no-analog plant communities (Gill et al. 2009, 2012). Additionally, rapid changes in climate during this time period led to dispersal lags in some European tree species (Svenning and Skov 2005, 2007), potentially creating transient species associations. In eastern North America, the influence of climate on fossil pollen assemblages was lower during the late Pleistocene than during the Holocene, indicating that biotic interactions or dispersal limitation may have been relatively more important (Blois et al. 2013a). In sum, environmental filtering, dispersal limitation, and biotic interactions all likely contributed to structuring fossil pollen taxa and assemblages across the past $21000 \mathrm{yr}$, but the effects of each process have not been explicitly teased apart.

In this paper, we classify which associations among pollen taxa are likely to be signals of direct biotic interactions versus indirect associations due to dispersal limitation or environmental filtering. To do this, we 1) determine with pairwise null model analysis (Gotelli and Ulrich 2010) which taxon associations are significant across space and whether these associations are persistent through time, and 2) develop methods to tease apart the relative influences of climate, dispersal limitation, and biotic interactions in explaining those associations. We first illustrate the method for a hypothetical presence-absence data matrix for a single time point. We then use paired fossil pollen and paleoclimate datasets from the last $21000 \mathrm{yr}$ in eastern North America to test this approach and examine changes in taxon associations through time. This framework applies to any paired datasets of species presence-absence and environmental characteristics at any time scale, whether paleontological or not. 


\section{Methods}

\section{Theoretical framework}

\section{Null model analyses of species co-occurrence}

A large literature on null model analyses of species cooccurrence has accumulated over the past $80 \mathrm{yr}$ (Harvey et al. 1983, Gotelli and Graves 1996). The initial impetus for these analyses was to ask whether co-occurrence patterns in replicated local assemblages were any different than might be expected by chance (Connor and Simberloff 1979). Deviations from this simple null hypothesis were thought to reflect species interactions (primarily interspecific competition), but might also reflect environmental filtering or dispersal limitation. More recent analyses have emphasized the contribution of individual species pairs to assemblage-level patterns of species co-occurrence (Cardillo and Meijaard 2010). The typical dataset for a null model analysis is organized into a binary presence-absence matrix (McCoy and Heck 1987) in which rows are taxa, columns are sites or samples, and entries indicate the presence (1) or absence (0) of a particular species in a site. These matrices are commonly collected by community ecologists who sample contemporary assemblages, but replicated fossil data based on standardized sampling methods can be represented and analyzed in exactly the same way.

\section{Quantifying species co-occurrence}

The strength of association between two species can be quantified by the C-score (Stone and Roberts 1990). For a particular pair of species in the matrix, the $\mathrm{C}$-score is calculated as $C_{a b}=\left(R_{a}-S\right)\left(R_{b}-S\right)$. Here $R_{a}$ is the row total for species $a$ (number of occurrences of species $a$ ), $R_{b}$ is the row total for species $b$, and $S$ is the number of sites that contain both species $a$ and species $b$. Relatively large values of $C_{a b}$ indicate that the species pair is segregated, with few or no sites that contain representatives of both species. Relatively small values of $C_{a b}$ indicate that the species pair is aggregated or nested, with many sites containing representatives of both species. The minimum value of $C_{a b}$ is zero, which occurs when at least one species always co-occurs with the other $\left(R_{a}=S\right.$ or $\left.R_{b}=S\right)$. The maximum value of $C_{a b}$ is $\left(R_{a}\right)\left(R_{b}\right)$, which occurs when both species never co-occur $(S=0)$. The average $C$-score calculated for all possible species pairs has traditionally been used as a single metric of community-wide aggregation or segregation. However, a matrix with a significantly large $C$-score (segregated matrix) will contain both aggregated and segregated species pairs (Ulrich and Gotelli 2010). For this reason, we first tested each data matrix for significance of the overall $C$-score, and then proceeded to determine which individual species pairs were significantly aggregated or segregated.

\section{Null models}

$C$-scores for individual species pairs were compared to the statistical expectation for a set of stochastic matrices in which species interactions, environmental associations, and dispersal limitations are not important. These matrices were not generated by a mechanistic model of species dispersal and colonization, such as the neutral model (Hubbell 2001). Instead, they were generated by a simple statistical randomization that captures the general effects of these processes without specifying a particular mechanistic model and its parameter values (Gotelli and Ulrich 2012). We used a 'fixed-fixed' null model (Gotelli 2000) in which the sample space consists of random matrices that have the same dimensions as the original matrix, the same percentage matrix fill, and the same row and column totals. The use of fixed row and column totals preserves inherent heterogeneity that is commonly observed in the number of species occurrences (row totals), and the number of species observed per site (column totals). Preserving these features of the data matrix is especially important for paleoecological matrices because taphonomic biases can cause some species to be preserved more often than others, and can cause some sites or samples to yield more species than others (Behrensmeyer et al. 2000). By preserving these features in the original data matrix, the fixed-fixed algorithm ensures that only patterns of species co-occurrence above and beyond those generated by the margin totals of the matrix will be detected. Extensive benchmark testing for co-occurrence analysis (Gotelli 2000) and nestedness analysis (Ulrich and Gotelli 2010) has demonstrated that the fixed-fixed algorithm has good type I error properties, and will infrequently reject the null hypotheses for heterogeneous matrices generated by simple stochastic sampling. At the same time, the fixed-fixed algorithm has reasonably good statistical power for detecting patterns of species segregation in structured matrices that have been deliberately seeded with random noise (Gotelli 2000).

We generated the $C$-score and implemented the fixed-fixed algorithm in the FORTRAN program Pairs (Ulrich 2008). Pairs uses an efficient swapping algorithm, in which the elements of $2 \times 2$ submatrices are randomly chosen and swapped. This algorithm creates a sequence of matrices that preserve row and column total sums. However, sequential matrices created by swapping this way are serially correlated and not truly independent. To break this dependence, we used an independent swap algorithm (Gotelli and Entsminger 2003), in which each random matrix is generated by a separate swapping sequence that always begins with the original matrix. To create each random matrix, we swapped $\mathrm{n}$ submatrices, where $\mathrm{n}$ equals 10 times the number of matrix elements (e.g. $10 \times$ (number of species $\times$ number of sites)). A fresh sequence of swaps was used to generate each random matrix. One thousand random matrices were created this way for each empirical matrix, although consistent results can be obtained with as few as 100 random matrices (Ulrich 2008).

\section{Testing individual pairs}

In a standard null model analysis, a single community-wide metric (such as the average $C$-score for a matrix) is compared to the histogram of $C$-scores generated for a sample of random matrices $\left(\mathrm{H}_{\mathrm{o}}\right)$, and the probability of the observed $C$-score $\mid \mathrm{H}_{\mathrm{o}}$ is estimated from the tail area of the simulated distribution (Manly 1995). The same approach to estimating $\mathrm{p}$ values can be applied to the $C$-score measured for each individual pair of species in the matrix. However, because there are $m(m-1) / 2$ such non-independent pairs of species in a single matrix (where $m=$ number of species in the matrix), the potential rate of false positives with so 
many statistical tests is quite high. A similar problem arises in the analysis of microarrays, in which the expression levels of thousands of potentially non-independent genes are assayed with parametric or non-parametric statistical tests (Kammenga et al. 2007). For null model analysis of the cooccurrence of individual species pairs (Gotelli and Ulrich 2010), we adapted an empirical Bayes approach originally proposed by Efron (2005) for this problem of screening large numbers of non-independent tests. In brief, $C$-scores for each species pair are rescaled to a $[0,1]$ range and binned into histogram categories. Next, the simulated data are binned in a similar way, and the mean and $95 \%$ confidence interval of the $C$-scores of simulated species pairs in each bin is calculated. Finally, the original $C$-score values within each bin are ordered from smallest to largest $C$-scores. For the Pairs analysis, pairs of species are retained whose $C$-scores are above the simulated mean for the bin (Bayesian mean criterion), and which would be statistically significant if the species pair was treated as an independent test. This 'double screen' reduces some of the false positives that would arise by simply retaining all species pairs for which the uncorrected association (aggregated or segregated) yielded $\mathrm{p}<0.05$. For the bins that are near 0.0 , these largest $C$ scores will represent aggregated species pairs. For the bins that are near 1.0, these largest $C$ scores will represent segregated species pairs. This is less conservative than a cut-point based on the $95 \%$ confidence interval for bin deviations, but more conservative than an unadjusted count of significant pairs, and usually more conservative than a sequential Bonferroni correction in which the pairs are ordered by their $\mathrm{p}$ values and a cutoff is imposed that is determined by both the individual $p$-value and its rank. Benchmark tests of the Pairs algorithm show that it is effective (though not perfect) at controlling for false positives while still allowing for detection of a relatively small subset of non-random species pairs from a binary presence-absence matrix (Gotelli and Ulrich 2010). We ran the Pairs analysis for each data matrix to identify the subset of species pairs that exhibited strong aggregation or segregation.

\section{Identifying the causes of non-randomness}

Null model analysis has been a successful tool for identifying non-random patterns of species associations. But the analysis cannot, by itself, point to the causes of such segregated or aggregated patterns. Here we consider explicitly two major classes of mechanisms that might lead to non-random associations of species pairs: dispersal limitations and habitat or climate (environmental) filtering of species into groups with similar environmental niches. All significant pairs that did not show signals of significant environmental variation or dispersal limitation may provide evidence of a significant species interaction, though it is also possible that environmental factors not considered in this analysis could contribute to nonrandom associations. To infer the roles of environmental factors and dispersal limitation, we move beyond the results of the standard null model tests with additional analysis of the characteristics of the sites. As described below, we focus specifically on subsets of sites that differ significantly in either environmental characteristics or spatial location.

\section{Site classifications}

For any particular pair of non-random species, each site can be assigned to one of four mutually exclusive classes, based on the presence of one species $(1,0)$ or $(0,1)$, both species $(1,1)$ or neither $(0,0)$ (Fig. 1$)$. The average characteristics of sites assigned to these 4 classes will generate additional patterns that can be used to distinguish among different causes of non-randomness. If the site characteristics are measured as continuous variables (such as average annual precipitation), sets of sites can be compared with ANOVA or t-tests. If the site characteristics are measured as discrete variables (such as depositional environment for fossil materials), sets of sites can be compared with a 2-way contingency table analysis. In each case, the analysis will pinpoint whether characteristics of sites vary systematically based on the presence or absence of each species member in the pair.

For segregated species pairs, the critical comparison is between sites that have one species $(1,0)$ and sites that have the other species $(0,1)$ (i.e. allotopic sites). If species interactions are the critical factor in producing segregation, these two classes of allotopic sites should not differ systematically in either their environmental characteristics or their spatial arrangement. Such species interactions might include pairwise competition or predation, but also might reflect indirect effects of other species. For aggregated species pairs, the critical comparison is between sites that have both species $(1,1)$ (i.e. syntopic sites) and sites that have neither species $(0,0)$. If species interactions are important in producing aggregation, these syntopic and empty sites should not differ systematically in either their environmental characteristics or their spatial arrangement. Such pairwise interactions might include pairwise mutualism or commensalism (or even predation), but also might reflect indirect effects of other species.

\section{Environment tests}

For each site within a data matrix, we have different measures of environment, either continuous (e.g. annual precipitation or temperature, as in this study; see Climate and distance data) or categorical (e.g. soil type or depositional environment). In the case of continuous measures, a one-way ANOVA can be used to compare the environment between the allotopic sites of segregated pairs $((1,0)$ vs $(0,1))$ and between the syntopic sites and empty sites of aggregated pairs $((1,1)$ vs $(0,0))$. The null hypothesis is that site characteristics do not differ systematically between these pairs of site classifications. For the categorical measures, a two-way contingency table can be used to classify the sites. For the segregated species pairs, we counted the frequency of each environmental type for the two kinds of allotopic sites $((1,0)$ and $(0,1))$. For the aggregated species pairs, we counted the frequency of each environmental type for the syntopic sites $(1,1)$ and the empty sites $(0,0)$. For both kinds of two-way data tables, we used a chi-square test of association. The null hypothesis was that the frequencies of different environmental types did not differ among the site classes. If this null hypothesis is rejected, a parsimonious interpretation is that environmental associations are at least partly responsible for segregated or aggregated patterns of species occurrence (Fig. 1, 2). 
Is the species pair significantly associated?
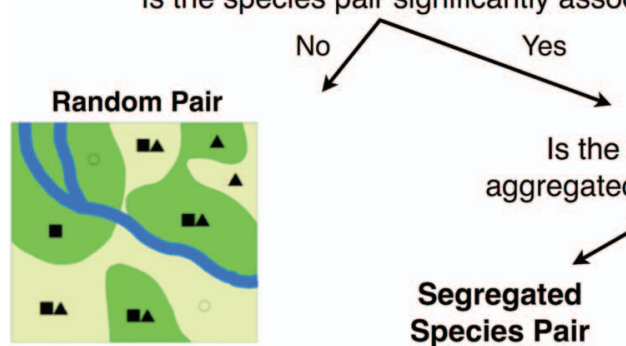

Is the species pair aggregated or segregated?
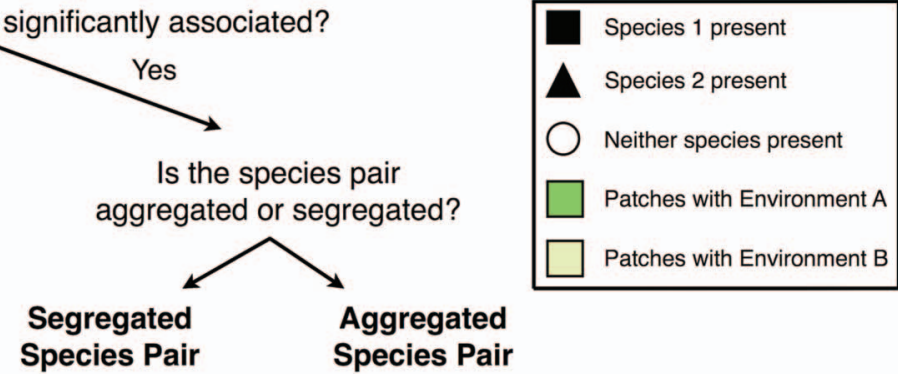
Species Pair

\section{Species Pair}

Is the environment at 10 vs 01

Is the environment at 00 vs 11 sites significantly different?

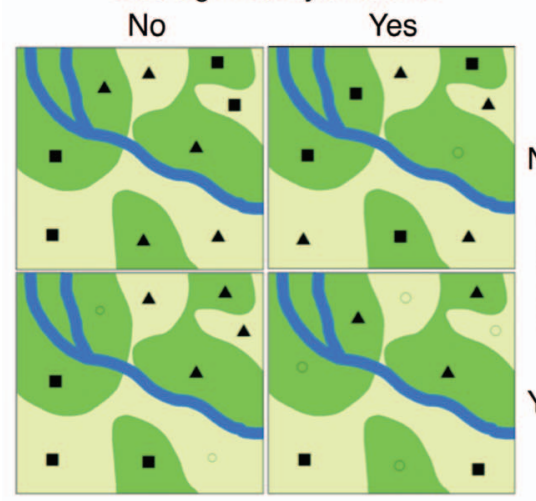

No

sites significantly different?

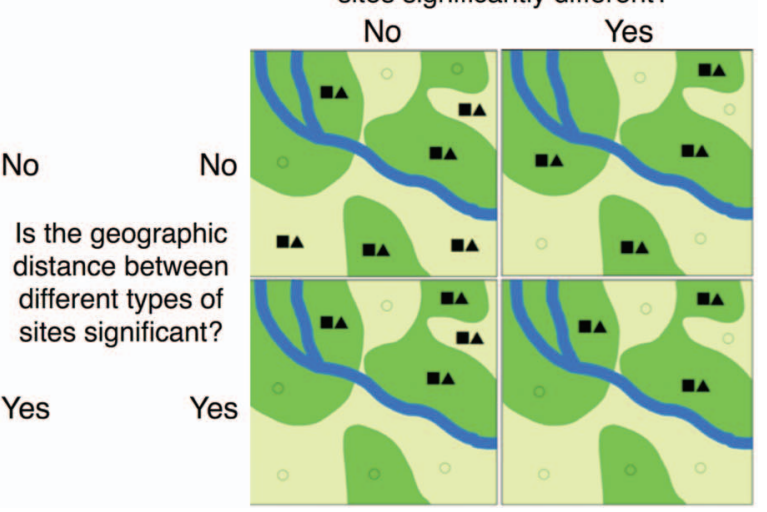

Figure 1. Hypothetical patterns of species associations on the landscape under nine scenarios.

Is the species pair significantly associated?

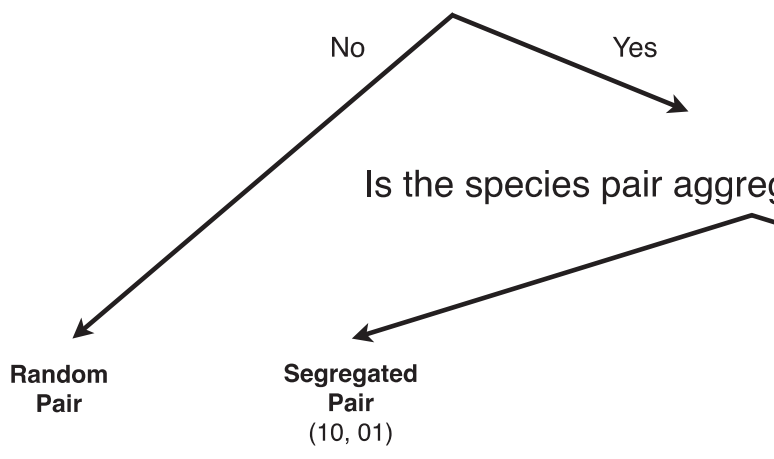

Is the geographic distance between different types of sites significant?

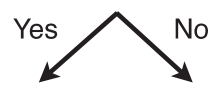

Is the climate at different types of sites significant?

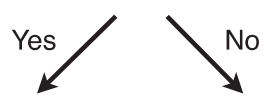

Is the climate at different types of sites significant?
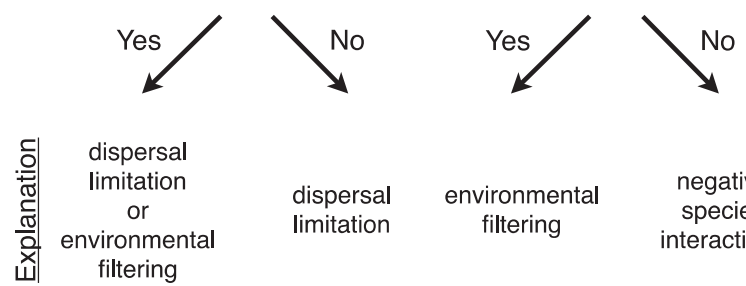

dispersal limitation
environmental filtering

Figure 2. Decision tree showing ecological explanations for the outcome of tests for the causes of significantly segregated or aggregated pairs. 


\section{Spatial overlap}

In addition to environmental characteristics of the sites, we also often have geographic coordinates for each site. We treated the geographic coordinates as a two-element response vector, and then used a one-way MANOVA to compare the distance in coordinate space between the group centroids of the different site types (e.g. Supplementary material Appendix 1, Fig. A1). Note that this analysis does not simply compare the geographic separation of sites with species A versus species B. Rather, for segregated pairs, it tests for the spatial overlap of allotopic sites $((1,0)$ and $(0,1))$, and, for aggregated pairs, it tests for the spatial overlap of syntopic $(1,1)$ and empty $(0,0)$ sites. The null hypothesis is that the different classes of sites are not spatially segregated from one another. If this null hypothesis is rejected for segregated pairs, a parsimonious interpretation is that some form of historical dispersal limitation is at least partly responsible for the pattern of aggregated or segregated occurrence of a particular species pair. We decided to not impose a false positive correction to the set of spatial overlap and environmental tests, because we were already working with a subset of species pairs that are non-random, and did not wish to be overly conservative. However, this decision does affect the outcome of the classification scheme (see Discussion).

\section{Logical outcomes}

The above framework leads to a forked logic tree with a total of 9 possible outcomes (Fig. 2). The first level of branching is a tripartite division of all species pairs into random, aggregated, or segregated. Within the aggregated and segregated pairs, each fork can be further divided by whether or not sites show significant patterns of spatial proximity, and then further divided by whether or not sites differ in environmental characteristics (or vice versa; the order of division after the first level of branching does not matter). The logic outcomes in the tree are not mutually exclusive. For example, ecologists working in the MacArthurian tradition would argue that competitive interactions can force inferior competitors into substandard environments and reduce spatial overlap, and that these kinds of interactions can scale up to the biogeographic scale. But for fossil data, whose total length spans timescales of centuries to millennia and beyond (and a temporal resolution ranging from decadal to multi-centennial or longer), it is more parsimonious to attribute such patterns to differences in environment, depositional environment, or dispersal limitation, if there is evidence for these processes. Thus, in this framework, species interactions are invoked only for cases in which species pairs are significantly aggregated or segregated, but there is no evidence of environmental associations or non-random spatial associations (Fig. 2).

\section{Data and quantitative analysis}

\section{Fossil pollen data}

To test our framework with paleontological data, we relied on fossil pollen data from the late Quaternary of eastern North America, which provide a spatially averaged description of vegetation composition with a resolution that can vary from ca $10 \mathrm{~m}$ to $10 \mathrm{~km}$, depending on the site and vegetation (Prentice 1988, Webb 1993). These data have been used previously for biogeographic analyses of community dissimi- larity (Blois et al. 2013a, b). Briefly, the fossil pollen data are drawn from sites in the Neotoma Paleoecology Database (<www.neotomadb.org $>$ ), with site age models revised and standardized (Blois et al. 2011). Previous work indicates that the absolute temporal precision among sites in the dataset is about $500 \mathrm{yr}$ throughout the latest Pleistocene, although relative temporal precision within sites can be on the order of decades to centuries (Blois et al. 2011). Thus, the data allow confident inferences of ecological responses to millennialscale climate change despite uncertainty in the base data. Overall, 527 sites contributed to the dataset, though not all sites were included at all times (Supplementary material Appendix 1, Fig. A2). Most sites were lacustrine environments, and the majority of the remaining sites were bogs or mires. Full details of site selection are given in Blois et al. (2011). We use the term 'taxon associations' throughout the paper when referring to our pollen data because taxonomic resolution in this paper is usually but not strictly at the genus level. Many pollen types naturally correspond to genus resolution, though in some cases the taxa represent closely related and palynologically indistinguishable genera (Ostrya and Carpinus, Juniperus and Thuja, Rumex and Oxyria, and Ambrosia-type pollen grains). Any available species-level data were aggregated to the generic level. The original fossil pollen data represent the relative abundance of each fossil pollen taxon at each site, calculated relative to the total upland pollen sum for the site, which included taxa identified to taxonomic levels higher than genus. Relative abundances were then linearly interpolated to 1000 -yr time steps, for every $1000 \mathrm{yr}$ from 21 to 0 thousands of calibrated years before present (kyr BP). The program Pairs (Ulrich 2008), used for later analyses, considers any value greater than zero as 'present'. This conversion may classify some taxa that are particularly prone to long-distance transport (e.g. Pinus) as 'present' when they were not actually present in the plant community surrounding the lake (Webb et al. 1981). Additionally, some of the rare taxa may be classified as absent when they were present on the landscape. Thus, the inferred number of significant taxonomic associations is likely to be an underrepresentation of the actual number of significant pairs through time. In sum, the base data consist of a presence-absence matrix for all 106 fossil pollen taxa at all sites across eastern North America, for a snapshot of time every $1000 \mathrm{yr}$ from $21 \mathrm{kyr} \mathrm{BP}$ to the present. These 22 presence-absence matrices (rows $=$ genera, columns $=$ sites) and 22 site-environment matrices (rows $=$ sites, columns $=$ environment, described below) have been deposited in FigShare (doi: 10.6084/m9.figshare.841756).

\section{Climate and distance data}

To quantify environmental characteristics at each site, we relied on paleoclimate simulations from the National Center for Atmospheric Research Community Climate System Model ver. 3 (CCSM3) developed by Liu et al. (2009). CCSM3 provides transient paleoclimate simulations that are independent of the pollen data starting at $22 \mathrm{kyr} \mathrm{BP}$, with seasonally averaged model outputs saved at a decadal time step (Liu et al. 2009). These data were downscaled to a $0.5 \times 0.5$ degree grid by Veloz et al. (2012) for every $1000 \mathrm{yr}$ from $21 \mathrm{kyr}$ BP to the present (available at $<$ purl. org/climate $>$ ). For each site and available time slice, we 
extracted the mean annual precipitation, mean winter (December-February) temperature, and mean summer (June-August) temperature. Because our primary concern was teasing apart the relative influence of climate or dispersal limitation on generating significant taxon associations rather than looking at the influence of individual climate variables on pairwise associations, the three variables were first scaled and centered, and then transformed to a principal components axis (PCA). We used the scores from the first two principal components as measures of 'climate' at each site. The first principal components axis explained from $65.7-84.1 \%$ of the variation, depending on the time period (Supplementary material Appendix 1, Table A1). While axis 1 had the strongest correlations with winter temperature, all three climate variables (precipitation, winter temperature, summer temperature) loaded relatively evenly at all time points (Supplementary material Appendix 1, Table A1). The second principal components axis primarily captured variation in precipitation and secondarily variation in summer temperature, and explained $13.9-31.3 \%$ of the variation. Together, the first two axes explain $93.7-98.3 \%$ of the total climate variation and thus represent suitable proxies for the overall 'climate' experienced at each site through time. Geographic coordinates are in North America Albers Equal Area Conic projection (origin: $40 \mathrm{~N}, 96 \mathrm{~W}$, standard parallels: $20 \mathrm{~N}, 60 \mathrm{~N}$ ).

\section{Pairs analysis}

We used the program Pairs (Ulrich 2008) to detect significant positive and negative taxon associations across space for each time period separately, implementing the Bayesian mean criterion for assessing significance as described above.

\section{Environment and dispersal analyses}

Because climate variables were continuous, we used a MANOVA with the first two principal component scores for each site as a two-element response vector to test for the influence of climate between the allotopic sites of segregated pairs $((1,0)$ vs $(0,1))$ and between the syntopic and empty sites of aggregated pairs $((1,1)$ vs $(0,0))$. Similarly, we used a MANOVA test with the geographic coordinates of each site to test for dispersal limitation, as described above. In this case, the test boils down to whether the geographic centroid of sites in one category (e.g. $(1,0)$ for segregated pairs) is significantly different from the geographic centroid of sites in the other category (e.g. $(0,1)$ for segregated pairs) (Supplementary material Appendix 1, Fig. A1). We then tallied the number and proportion of aggregated pairs and segregated pairs in four categories: 1) taxon pairs that show a signal of dispersal limitation (significant value for the distance test); 2) taxon pairs that show a signal of climatic differences (significant value for environment test); 3) taxon pairs that show signals of both dispersal limitation and climatic differences; and 4) taxon pairs that show signals of neither process. This final category represents cases in which taxon associations can be more confidently attributed to biotic interactions because there is no evidence of climatic effects and/or dispersal limitation.

\section{Results}

\section{Pairs}

Over the past $21000 \mathrm{yr}$, the number of unique taxon pairs ranged from 1176 (at $21 \mathrm{kyr} \mathrm{BP)} \mathrm{to} 3570$ (since $2 \mathrm{kyr} \mathrm{BP}$ ).

Table 1. Summary statistics for the Pairs analyses through time. From left to right, the columns show the time period of analysis (in thousands of calibrated years before present, kyr BP), number of sites in the dataset, the total number of taxon pairs, the proportion of random taxon pairs (relative to the total), the number of significantly segregated and aggregated taxon pairs, and the proportion of segregated and aggregated pairs, relative to the total number of non-random pairwise associations.

\begin{tabular}{|c|c|c|c|c|c|c|c|c|}
\hline $\begin{array}{l}\text { Age } \\
\text { (kyr BP) }\end{array}$ & $\begin{array}{l}\text { Number } \\
\text { of sites }\end{array}$ & $\begin{array}{l}\text { Total number } \\
\text { of pairs }\end{array}$ & $\begin{array}{c}\text { Number } \\
\text { random pairs }\end{array}$ & $\begin{array}{c}\text { Proportion } \\
\text { random pairs }\end{array}$ & $\begin{array}{c}\text { Number } \\
\text { segregated pairs }\end{array}$ & $\begin{array}{c}\text { Number } \\
\text { aggregated pairs }\end{array}$ & $\begin{array}{l}\text { Proportion } \\
\text { segregated }\end{array}$ & $\begin{array}{l}\text { Proportion } \\
\text { aggregated }\end{array}$ \\
\hline 0 & 233 & 3570 & 3501 & 0.9807 & 48 & 21 & 0.6957 & 0.3043 \\
\hline 1 & 422 & 3570 & 3522 & 0.9866 & 21 & 27 & 0.4375 & 0.5625 \\
\hline 2 & 418 & 3570 & 3541 & 0.9919 & 10 & 19 & 0.3448 & 0.6552 \\
\hline 3 & 393 & 3321 & 3286 & 0.9895 & 14 & 21 & 0.4000 & 0.6000 \\
\hline 4 & 383 & 3321 & 3285 & 0.9892 & 9 & 27 & 0.2500 & 0.7500 \\
\hline 5 & 358 & 3240 & 3202 & 0.9883 & 13 & 25 & 0.3421 & 0.6579 \\
\hline 6 & 342 & 3486 & 3472 & 0.996 & 4 & 10 & 0.2857 & 0.7143 \\
\hline 7 & 323 & 3160 & 3141 & 0.994 & 3 & 16 & 0.1579 & 0.8421 \\
\hline 8 & 291 & 2926 & 2899 & 0.9908 & 2 & 25 & 0.0741 & 0.9259 \\
\hline 9 & 271 & 2926 & 2901 & 0.9915 & 7 & 18 & 0.2800 & 0.7200 \\
\hline 10 & 246 & 2556 & 2528 & 0.989 & 7 & 21 & 0.2500 & 0.7500 \\
\hline 11 & 199 & 2485 & 2466 & 0.9924 & 2 & 17 & 0.1053 & 0.8947 \\
\hline 12 & 149 & 2556 & 2542 & 0.9945 & 1 & 13 & 0.0714 & 0.9286 \\
\hline 13 & 111 & 2556 & 2542 & 0.9945 & 2 & 12 & 0.1429 & 0.8571 \\
\hline 14 & 72 & 2211 & 2200 & 0.995 & 3 & 8 & 0.2727 & 0.7273 \\
\hline 15 & 49 & 1953 & 1946 & 0.9964 & 4 & 3 & 0.5714 & 0.4286 \\
\hline 16 & 39 & 1830 & 1826 & 0.9978 & 2 & 2 & 0.5000 & 0.5000 \\
\hline 17 & 30 & 1485 & 1480 & 0.9966 & 4 & 1 & 0.8000 & 0.2000 \\
\hline 18 & 21 & 1326 & 1324 & 0.9985 & 1 & 1 & 0.5000 & 0.5000 \\
\hline 19 & 16 & 1485 & 1484 & 0.9993 & 0 & 1 & 0.0000 & 1.0000 \\
\hline 20 & 15 & 1485 & 1481 & 0.9973 & 4 & 0 & 1.0000 & 0.0000 \\
\hline 21 & 13 & 1176 & 1176 & 1 & 0 & 0 & 0.0000 & 0.0000 \\
\hline Total & & 56194 & 55745 & & 161 & 288 & & \\
\hline
\end{tabular}



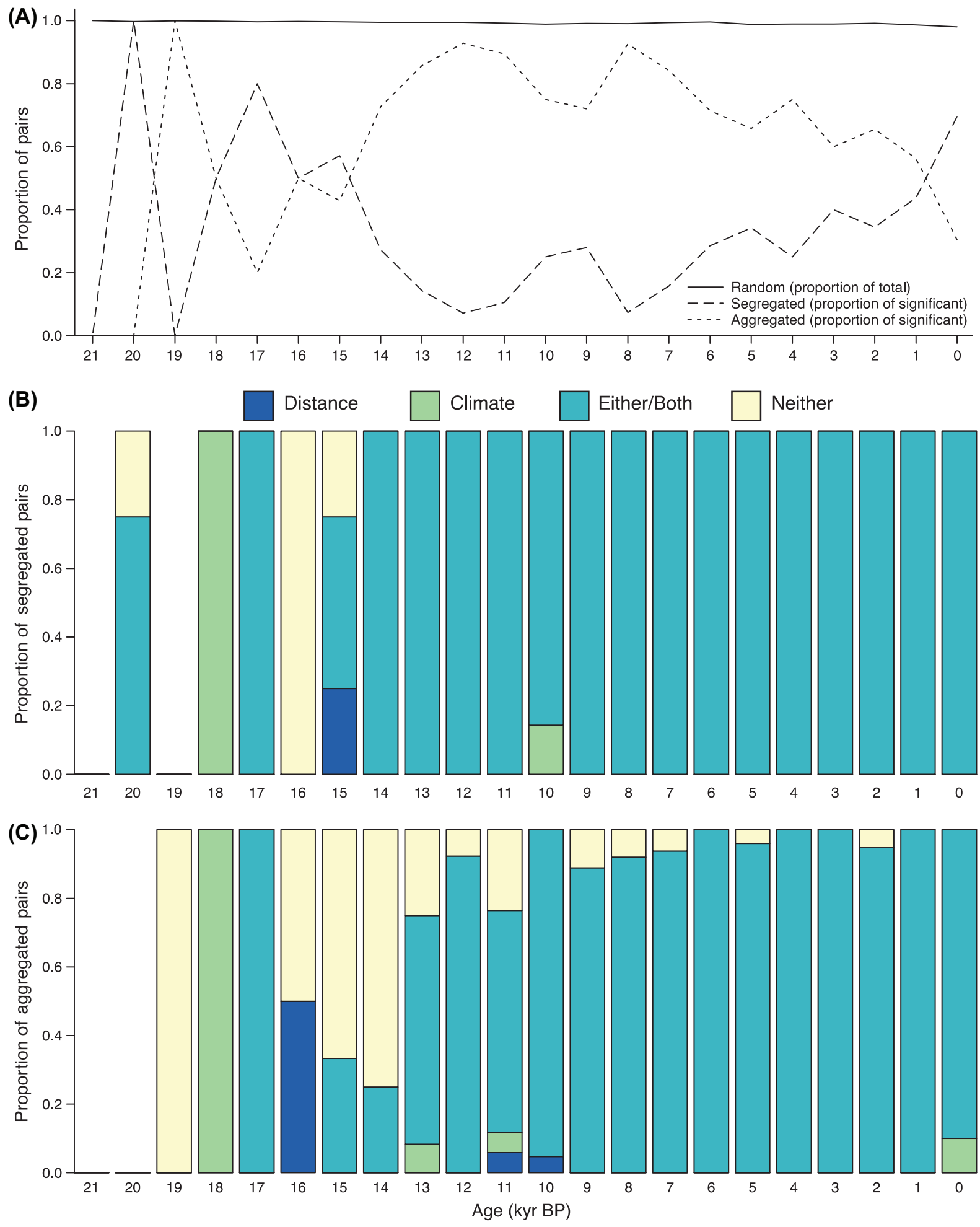

Figure 3. (A) Proportion of random (relative to the total number of pairs; solid line along the top of the graph), segregated, and aggregated taxon pairs from $21000 \mathrm{yr}$ to the present. The proportion of segregated and aggregated pairs is calculated relative to the proportion of significant pairs. See Table 1 for sample sizes for each time period. (B) Attribution of the causes of significantly segregated pairs, e.g. the relative proportion of significantly segregated pairs that can be attributed to climate (blue), dispersal limitation (red), either or both processes (purple), or neither climate nor dispersal processes (e.g. a potential biotic interaction; gray). (C) same as (B), but for aggregated pairs.

In all time periods, nearly all taxon pairs were randomly associated (98 to $100 \%$ of possible taxon pairs; Table 1, Fig. 3), reflecting the conservative nature of the Pairs tests for significantly associated taxon pairs. Of the non-random taxon pairs, the proportion of significantly segregated versus aggregated pairs varied through time. For most of the past $21000 \mathrm{yr}$, the aggregated pairs were more frequent than segregated pairs, but the proportion of segregated pairs steadily increased through 
Table 2. The proportion of segregated and aggregated pairs that can be attributed to climate, dispersal limitation, both, or neither process through time. Note that the total number of segregated or aggregated pairs per time slice may be different than the totals in Table 1 because statistical significance could not be assessed for some taxon pairs.

\begin{tabular}{|c|c|c|c|c|c|c|c|c|}
\hline \multirow{2}{*}{$\begin{array}{l}\text { Age } \\
(\text { kyr BP) }\end{array}$} & \multicolumn{4}{|c|}{ Proportion of segregated pairs } & \multicolumn{4}{|c|}{ Proportion of aggregated pairs } \\
\hline & Distance & Climate & Both & Neither & Distance & Climate & Both & Neither \\
\hline 0 & 0 & 0 & 1 & 0 & 0 & 0.1 & 0.9 & 0 \\
\hline 1 & 0 & 0 & 1 & 0 & 0 & 0 & 1 & 0 \\
\hline 2 & 0 & 0 & 1 & 0 & 0 & 0 & 0.9474 & 0.0526 \\
\hline 3 & 0 & 0 & 1 & 0 & 0 & 0 & 1 & 0 \\
\hline 4 & 0 & 0 & 1 & 0 & 0 & 0 & 1 & 0 \\
\hline 5 & 0 & 0 & 1 & 0 & 0 & 0 & 0.96 & 0.04 \\
\hline 6 & 0 & 0 & 1 & 0 & 0 & 0 & 1 & 0 \\
\hline 7 & 0 & 0 & 1 & 0 & 0 & 0 & 0.9375 & 0.0625 \\
\hline 8 & 0 & 0 & 1 & 0 & 0 & 0 & 0.92 & 0.08 \\
\hline 9 & 0 & 0 & 1 & 0 & 0 & 0 & 0.8889 & 0.1111 \\
\hline 10 & 0 & 0.1429 & 0.8571 & 0 & 0.0476 & 0 & 0.9524 & 0 \\
\hline 11 & 0 & 0 & 1 & 0 & 0.0588 & 0.0588 & 0.6471 & 0.2353 \\
\hline 12 & 0 & 0 & 1 & 0 & 0 & 0 & 0.9231 & 0.0769 \\
\hline 13 & 0 & 0 & 1 & 0 & 0 & 0.0833 & 0.6667 & 0.25 \\
\hline 14 & 0 & 0 & 1 & 0 & 0 & 0 & 0.25 & 0.75 \\
\hline 15 & 0.25 & 0 & 0.5 & 0.25 & 0 & 0 & 0.3333 & 0.6667 \\
\hline 16 & 0 & 0 & 0 & 1 & 0.5 & 0 & 0 & 0.5 \\
\hline 17 & 0 & 0 & 1 & 0 & 0 & 0 & 1 & 0 \\
\hline 18 & 0 & 1 & 0 & 0 & 0 & 1 & 0 & 0 \\
\hline 19 & 0 & 0 & 0 & 0 & 0 & 0 & 0 & 1 \\
\hline 20 & 0 & 0 & 0.75 & 0.25 & 0 & 0 & 0 & 0 \\
\hline 21 & 0 & 0 & 0 & 0 & 0 & 0 & 0 & 0 \\
\hline
\end{tabular}

time, particularly from $8 \mathrm{kyr}$ BP (7\% segregated pairs) to the present (70\% segregated pairs) (Table 1, Fig. 3).

\section{Environment and dispersal analyses}

The vast majority of sites with significantly aggregated or segregated taxon pairs differed in climate or geographic distance, and usually differed in both aspects (Table 2, Fig. 3). There were only a few cases in which neither climate nor geographic differences among sites were detected for significant taxon associations. In such cases, we infer that biotic interactions may have played a role. The proportion of aggregated pairs that were potentially caused by biotic interactions increased further back in time (Table 2, 3, Fig. $3 \mathrm{~b}, \mathrm{c})$, indicating that the effect of biotic interactions may have been more important in the latest Pleistocene, during deglaciation, than in the Holocene. Most taxon pairs classified as potential examples of biotic interactions appeared during one time period only, with the exception of Bidens and Xanthium (14-11 kyr BP), Vaccinium and Chamaedaphne (5 and 2 kyr BP), and Sambucus and Hypericum (11 and 8 kyr BP) (Table 3).

\section{Discussion}

Environmental filtering, dispersal limitation, and biotic interactions are three intertwined processes that have been commonly invoked to explain patterns of species associations across the landscape and through time (Tuomisto et al. 2003, Kraft et al. 2008, Gotelli et al. 2010). They are often examined separately (but see Jiménez et al. 2012), however, and no unified framework exists for disentangling the three processes. Here, we provide such a framework and examine the role of these three processes in explaining spatial patterns of taxon associations through time for late Quaternary fossil pollen assemblages. While it may still be impossible to cleanly disentangle processes (e.g. when signals are consistent with both dispersal limitation and environmental filtering), our framework narrows down the sets of taxa for which biotic interactions are most likely to be important and leads to an explicit consideration of the kinds of patterns that can be used to distinguish environmental correlates from the effects of biotic interactions or dispersal limitation (Fig. 1, 2).

Within the subset of 449 significant pairs, we found that the potential role of biotic interactions in explaining significant taxon associations at these spatial and temporal scales was minimal. Instead, the correlational patterns reported here are most consistent with climatic filtering or dispersal constraints across space as the key processes controlling generic coexistence of pollen taxa in eastern North America appear to be either climatic filtering or dispersal constraints across space; most often both processes were consistent with the data (Fig. 3). These two processes have been commonly invoked to explain patterns of community structure or species changes (Svenning and Skov 2007, Blois et al. 2013a) through time. The pairwise approach taken here emphasizes that pairwise associations detected in assemblages of species may be transitory and are often tied to climatic or environmental differences, or spatial effects.

\section{Patterns of significant pairs through time}

From $21 \mathrm{kyr}$ BP to the present, between 98 and 100\% of taxon pairs were randomly associated among sites (Table 1 , Fig. 3). The large proportion of random taxon pairs is 


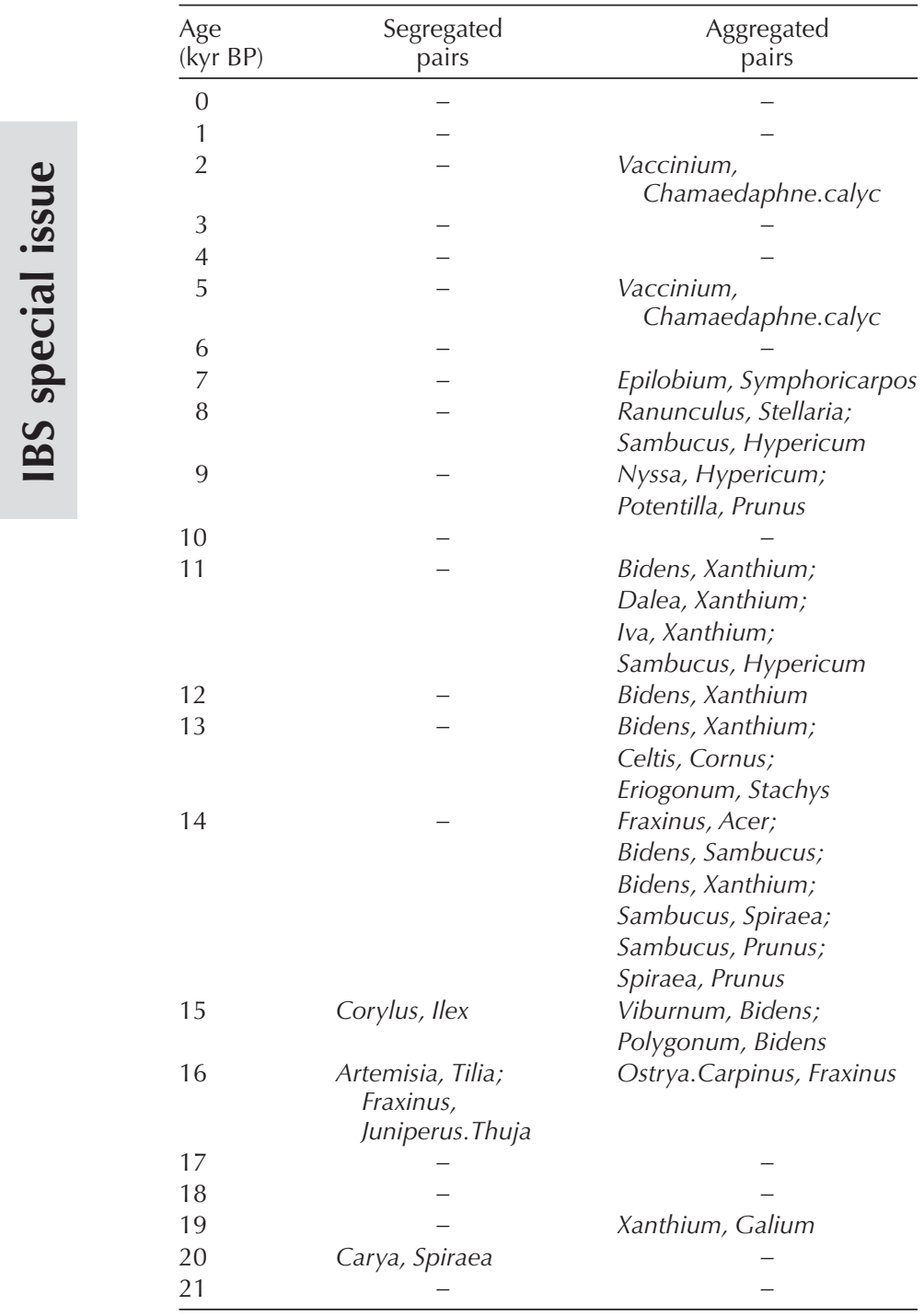

Table 3. The taxon pairs involved in significant segregations or aggregations through time.

unsurprising. The method of testing individual pairs imposes a strong screen for type I error, but more importantly, most taxa occur in relatively few sites. For good statistical reasons, it is difficult to assert that segregated pairs are non-random when both members of the pair are relatively rare, though we can detect aggregation more easily in this case. But without any additional evidence, the most parsimonious interpretation of the observation that two rare species do not co-occur frequently is that the pattern is due to chance. Thus, our approach is an inherently conservative method to begin with, but avoids falsely attributing biological processes to patterns that are more parsimoniously accounted for by simple sampling properties of the data.

Of the non-random subset, there were more aggregated than segregated pairs in most time periods (Table 1, Fig. 3). These results for fossil assemblages form an interesting contrast with a recent meta-analysis of pairwise associations in 272 presence-absence matrices for modern assemblages (Gotelli and Ulrich 2010). In modern assemblages, most species pairs also showed random associations, although these tended to be concentrated in data matrices from a relatively small number of studies. However, the nonrandom fraction for modern assemblages was dominated by segregated species pairs, with a 4-fold excess of perfectly segregated checkerboard pairs compared to the most conservative null expectation. In the fossil assemblages examined here, the segregated taxon pairs show a trend of increasing frequency over the last $8000 \mathrm{yr}$, but exceed the frequency of aggregated taxon pairs only in the most recent (modern) sample. One reason for the overall dominance of aggregated versus segregated taxon pairs in these data matrices (relative to modern data matrices) may be that our analyses were conducted at the genus level, which means that taxa are more widespread across space than the individual species within each genus and thus more likely to be aggregated. Additionally, differential species richness within genera may affect the patterns of aggregation and segregation, and sensitivity tests regarding the magnitude of this effect would be an interesting avenue for future research with modern data. Nevertheless, these explanations do not explain the trend through time in the numbers of segregated and aggregated taxon pairs. This time series of matrices contains a sample size bias, with number of sites increasing in more recent assemblages. With fewer sites there will typically be fewer taxa present and/or detected, which obviously affects the absolute number of significant taxon pairs that are identified. There is no obvious reason why differences in the number of sites should affect the relative frequency of segregated versus aggregated taxon pairs, however. One possibility is that this could relate to time-averaging within samples, especially if the older samples encompass more time than a sample taken from closer to the surface and thus artificially cause the appearance of aggregation. However, previous studies have shown that time-averaging is minimal in fossil sediment cores from lakes in eastern North America (Davis and Deevey 1964). Instead, the increasing proportion of segregated taxon pairs through time may also be a real pattern and reflect the stronger zonation of plant communities along latitudinal gradients during the Holocene compared to the late Pleistocene (Williams et al. 2004).

\section{Are significant taxonomic associations due to biotic interactions?}

The proportion of significantly segregated or aggregated pairs that were potentially caused by biotic interactions was higher in the latest Pleistocene, during deglaciation, than in the Holocene (Table 2). There were very few significant taxon pairs during this time to begin with (Supplementary material Appendix 1, Table A2), however. Additionally, Blois et al. (2013a) noted that the time slices 21-15 kyr BP were unreliable for inferring spatial dissimilarity patterns due to sample size differences through time (Table 1). Although there is no obvious reason that attribution of the cause of significant pairs would be biased by the low number of sites in the earlier times within the dataset, we regard the results from 21-15 kyr BP as tentative. If this is a real pattern, however, it could indicate increased importance of biotic interactions during a period of rapid environmental change and add to other work indicating that biotic interactions 


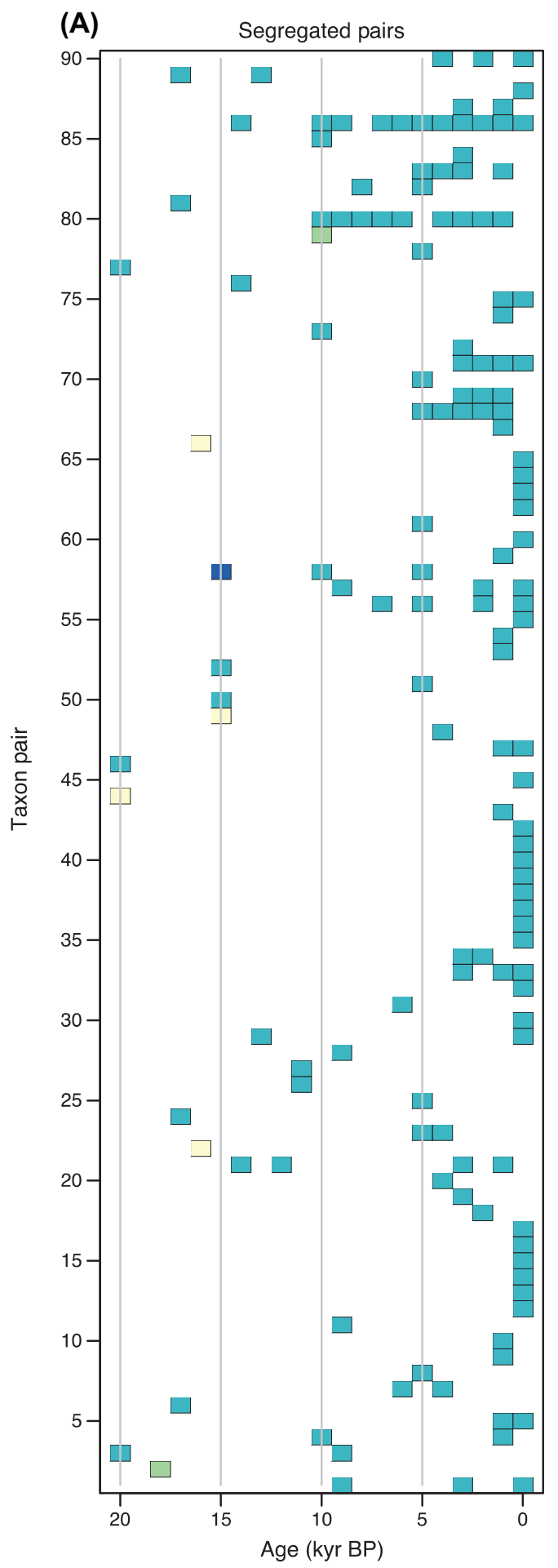

(B)

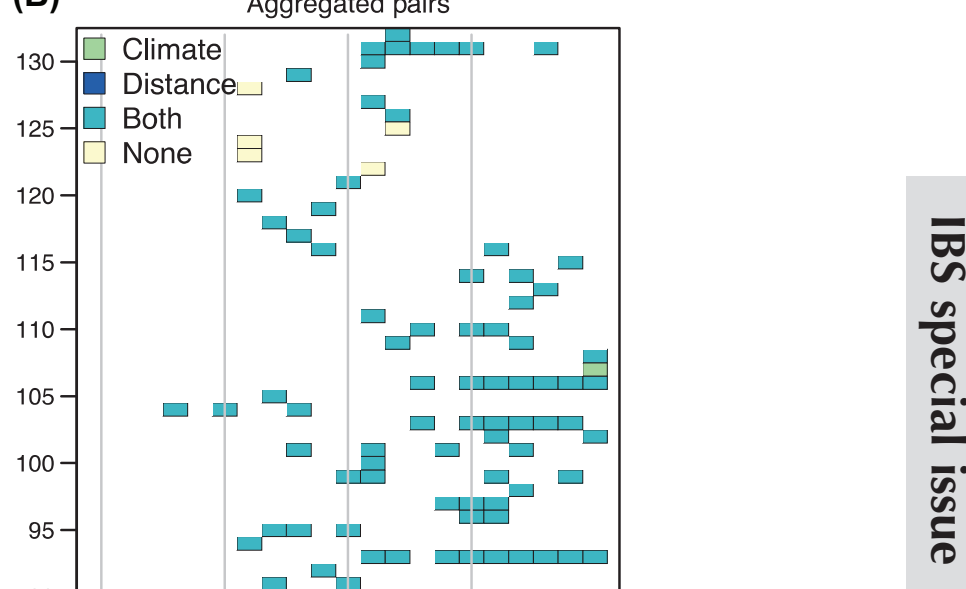

Figure 4. Patterns of attribution of the causes of a significant taxon pair through time, for (A) 161 segregated and (B) 288 aggregated pairs (Table 1). If there is no color for a given time period (e.g. whitespace), the taxon pair was classified as a random pair at that time or the cause could not be statistically attributed to any potential outcome. Colors are the same as in Fig. 3. See Supplementary material Appendix 1, Table A3 to match the index values with a taxon pair.

were significant drivers of community dynamics (Gill et al. 2009, 2012) under these circumstances.

Only three time periods showed significant taxon segregations that could not be attributed to either dispersal limitation or environmental differences. All of these were for time periods at or prior to $15 \mathrm{kyr} \mathrm{BP}-20,16$, and 15 kyr $\mathrm{BP}$ - and the segregations were transitory and involved different taxa in each case (Table 2, 3; Fig. 4A). If these associations do reflect the influence of biotic interactions, the interaction is transitory and does not persist through time. 
If we had imposed a false discovery screen for both the environmental and spatial overlap tests, we might have found more pairs that would be classified as examples of biotic interactions. However, most taxon pairs showed strong effects of both spatial and environmental segregation, so there would not be that many pairs classified as nonsignificant by both tests after screening for false positives.

Taxon aggregations were also infrequent, but more common than segregations. In 12 of 22 time slices there were taxon pairs for which aggregations could not be attributed to climate or large-scale spatial overlap between syntopic versus empty sites. These were most common between 16 and 11 kyr BP. Such pairs might reflect positive biotic interactions such as direct mutualisms, indirect effects such as shared pollinators or exclusion from the same sites due to a shared competitor or predator, or unmeasured habitat or climatic associations. The occurrence of potential positive biotic interactions in the latest Pleistocene could also provide support for the stress-gradient hypothesis, which posits that positive interactions buffer species in physically harsh environments (Bertness and Callaway 1994, He et al. 2013).

Similar to segregated pairs, many of the aggregated taxon pairs were impermanent through time. However, in the case of significantly aggregated pairs, particular taxa often were involved in significant aggregations at several time slices (Table 3). For example, Bidens and Xanthium were significantly aggregated through time, and those differences were not attributable to climate or distance, from 14-11 kyr BP. This association arises during the height of climate variability at the transition from glacial to interglacial periods, a time which also corresponds to the start of the 'no-analog' period at many of the sites in eastern North America (Gill et al. 2012). Neither genus is considered to be characteristic of the 'no-analog' period, however. Sample size is low for both Bidens and Xanthium (i.e. they are found at only a handful of sites in each time period), partly because both pollen types are often classified as undifferentiated Asteraceae. Taxonomic classification should be consistent throughout each individual core, so interanalyst and intersite variability in taxonomic identification should not bias one particular time period over another. Both genera often occur today in wetter sites, so they may prefer the same soils and hydrological environments (Chadde 2002) and this biotic association may reflect an unmeasured habitat association (such as soil type, which was not included as a habitat variable in this study because we do not have appropriate data for most sites and times). Life history characteristics rather than biotic interactions may also play a role - both genera contain zoochorous plants, so perhaps the same suite of dispersers was contributing to their aggregated pattern. For both potentially unmeasured habitat preferences and life history characteristics, it is unclear why the aggregation should arise only from 14-11 kyr BP. Later in the Holocene, the pair Vaccinium and Chamaedaphne (likely C. calyculata) was significantly aggregated at both 5 and $2 \mathrm{kyr}$ BP. Although our framework classifies this association as a biotic interaction, it more likely represents an unmeasured habitat association because many species in both Vaccinium and Chamaedaphne are found in peat bogs. Finally, the pair Sambucus and Hypericum represented a potential biotic interaction at two time slices (11 and $8 \mathrm{kyr} \mathrm{BP})$, although in this case there are no obvious unmeasured habitat characteristics or life history traits that explain the significant aggregation.

\section{Conclusions}

We have presented a parsimonious framework for analyzing species associations and inferring the roles of biotic interactions, habitat filtering, and dispersal limitation as causal agents. For fossil pollen assemblages of eastern North America, most taxon associations across space were random rather than significantly aggregated or segregated. The relatively few positive and negative taxon associations in space could be attributed to climatic filtering and/or dispersal limitation, and not to direct biotic interactions. For those rare taxon pairs that potentially represented biotic interactions, the interaction was not persistent through time. Our identification of biotic interactions is inherently conservative. Although environmental or geographic differences among sites were the primary processes associated with significant taxon associations, biotic interactions were likely operating as well; they simply were not the dominant drivers of associations at these scales of space and time. This suggests the possibility that species interactions may be more important in shaping community dynamics on short-term rather than paleoecological time scales. These methods can be applied to other assemblages of modern and fossil plants and animals for additional insights into the causes of species associations across a range of spatial and temporal scales.

Acknowledgements - Funding for this work was provided by the Smithsonian Inst. ETE program (publication \#292) and the National Science Foundation (DEB-1257625 awarded to SKL, AKB, and NJG and DEB-1257033 to JLB).

\section{References}

Ackerly, D. D. 2003. Community assembly, niche conservatism, and adaptive evolution in changing environments. - Int. J. Plant Sci. 164: S165-S184.

Baiser, B. et al. 2012. Geographic variation in network structure of a Nearctic aquatic food web. - Global Ecol. Biogeogr. 21: 579-591.

Bartlein, P. J. et al. 2011. Pollen-based continental climate reconstructions at 6 and 21 ka: a global synthesis. - Clim. Dyn. 37: 775-802.

Behrensmeyer, A. K. et al. 2000. Taphonomy and paleobiology. - Paleobiology 26: 103-147.

Bertness, M. D. and Callaway, R. 1994. Positive interactions in communities. - Trends Ecol. Evol. 9: 191-193.

Blois, J. L. et al. 2011. A methodological framework for assessing and reducing temporal uncertainty in paleovegetation mapping from late-Quaternary pollen records. - Quat. Sci. Rev. 30: 1926-1939.

Blois, J. L. et al. 2013a. Modeling the climatic drivers of spatial patterns in vegetation composition since the Last Glacial Maximum. - Ecography 36: 460-473.

Blois, J. L. et al. 2013b. Space can substitute for time in predicting climate-change effects on biodiversity. - Proc. Natl Acad. Sci. USA 110: 9374-9379.

Blois, J. L. et al. 2013c. Climate change and the past, present, and future of biotic interactions. - Science 341: 499-504. 
Cardillo, M. and Meijaard, E. 2010. Phylogeny and co-occurrence of mammal species on southeast Asian islands. - Global Ecol. Biogeogr. 19: 465-474.

Chadde, S. 2002. A Great Lakes Wetland flora: a complete guide to the aquatic and wetland plants of the Upper Midwest. - Pocketflora Press.

Collins, M. D. et al. 2011. Binary matrices and checkerboard distributions of birds in the Bismarck Archipelago. - J. Biogeogr. 38: 2373-2383.

Connor, E. F. and Simberloff, D. 1979. The assembly of species communities: chance or competition? - Ecology 60: 1132-1140.

Currano, E. D. et al. 2010. Fossil insect folivory tracks paleotemperature for six million years. - Ecol. Monogr. 80: 547-567.

Dalsgaard, B. et al. 2013. Historical climate-change influences modularity and nestedness of pollination networks. - Ecography 36: 1331-1340.

Davis, M. B. and Deevey, E. S. 1964. Pollen accumulation rates: estimates from Late-Glacial sediment of Rogers Lake. - Science 145: 1293-1295.

Efron, B. 2005. Bayesians, frequentists, and scientists. - J. Am. Stat. Assoc. 100: 1-5.

Gill, J. L. et al. 2009. Pleistocene megafaunal collapse, novel plant communities, and enhanced fire regimes in North America. - Science 326: 1100-1103.

Gill, J. L. et al. 2012. Climatic and megaherbivory controls on late-glacial vegetation dynamics: a new, high-resolution, multiproxy record from Silver Lake, Ohio. - Quat. Sci. Rev. 34: 66-80.

Gilman, S. E. et al. 2010. A framework for community interactions under climate change. - Trends Ecol. Evol. 25: 325-331.

Gotelli, N. J. 2000. Null model analysis of species co-occurrence patterns. - Ecology 81: 2606-2621.

Gotelli, N. J. and Graves, G. R. 1996. Null models in ecology. - Smithsonian Inst. Press.

Gotelli, N. J. and Entsminger, G. L. 2003. Swap algorithms in null model analysis. - Ecology 84: 532-535.

Gotelli, N. J. and Ulrich, W. 2010. The empirical Bayes approach as a tool to identify non-random species associations. - Oecologia 162: 463-477.

Gotelli, N. J. and Ulrich, W. 2012. Statistical challenges in null model analysis. - Oikos 121: 171-180.

Gotelli, N. J. et al. 2010. Macroecological signals of species interactions in the Danish avifauna. - Proc. Natl Acad. Sci. USA 107: 5030-5035.

Grimm, E. C. et al. 1993. A 50,000-year record of climate oscillations from Florida and its temporal correlation with the Heinrich events. - Science 261: 198-200.

Harvey, P. H. et al. 1983. Null models in ecology. - Annu. Rev. Ecol. Syst. 14: 189-211.

He, Q. et al. 2013. Global shifts towards positive species interactions with increasing environmental stress. - Ecol. Lett. 16: 695-706.

Hubbell, S. P. 2001. The unified neutral theory of biodiversity and biogeography (MPB-32). - Princeton Univ. Press.

Jablonski, D. 2008. Biotic interactions and macroevolution: extensions and mismatches across scales and levels. - Evolution 62: $715-739$.

Jiménez, J. J. et al. 2012. Soil environmental heterogeneity allows spatial co-occurrence of competitor earthworm species in a gallery forest of the Colombian 'Llanos'. - Oikos 121: 915-926.

Kammenga, J. E. et al. 2007. Microarray challenges in ecology. - Trends Ecol. Evol. 22: 273-279.

Kowalewski, M. 2002. The fossil record of predation: an overview of analytical methods. - Paleontol. Soc. Pap. 8: 3-42.
Kraft, N. J. B. et al. 2008. Functional traits and niche-based tree community assembly in an Amazonian forest. - Science 322: $580-582$.

Liu, Z. et al. 2009. Transient simulation of last deglaciation with a new mechanism for Bolling-Allerod warming. - Science 325: 310-314.

MacArthur, R. H. 1972. Geographical ecology. - Princeton Univ. Press.

Manly, B. F. 1995. A note on the analysis of species co-occurrences. - Ecology 76: 1109-1115.

McCoy, E. D. and Heck, K. L. Jr 1987. Some observations on the use of taxonomic similarity in large-scale biogeography. - J. Biogeogr. 14: 79-87.

Peńalver, E. et al. 2012. Thrips pollination of Mesozoic gymnosperms. - Proc. Natl Acad. Sci. USA 109: 8623-8628.

Phillips, O. L. et al. 2003. Habitat association among Amazonian tree species: a landscape-scale approach. - J. Ecol. 91: 757-775.

Prentice, I. C. 1988. Records of vegetation in time and space: the principles of pollen analysis. - In: Huntley, B. and Webb, T. III (eds), Vegetation history. Kluwer, pp. $14-72$.

Ricklefs, R. 2004. A comprehensive framework for global patterns in biodiversity. - Ecol. Lett. 7: 1-15.

Sfenthourakis, S. et al. 2006. Species co-occurrence: the case of congeneric species and a causal approach to patterns of species association. - Global Ecol. Biogeogr. 15: 39-49.

Shuman, B. N. et al. 2004. Evidence for the close climatic control of New England vegetation history. - Ecology 85: 1297-1310.

Stone, L. and Roberts, A. 1990. The checkerboard score and species distributions. - Oecologia 85: 74-79.

Svenning, J. and Skov, F. 2005. The relative roles of environment and history as controls of tree species composition and richness in Europe. - J. Biogeogr. 32: 1019-1033.

Svenning, J. and Skov, F. 2007. Could the tree diversity pattern in Europe be generated by postglacial dispersal limitation? - Ecol. Lett. 10: 453-460.

Tuomisto, H. et al. 2003. Dispersal, environment, and floristic variation of western Amazonian forests. - Science 299: 241-244.

Ulrich, W. 2008. Pairs - a FORTRAN program for studying pairwise species associations in ecological matrices. $-<$ www.uni. torun. $\mathrm{pl} / \sim$ ulrichw $>$.

Ulrich, W. and Gotelli, N. J. 2010. Null model analysis of species associations using abundance data. - Ecology 91: 3384-3397.

Ulrich, W. and Gotelli, N. J. 2012. A null model algorithm for presence-absence matrices based on proportional resampling. - Ecol. Model. 244: 20-27.

Veloz, S. D. et al. 2012. No-analog climates and shifting realized niches during the late Quaternary: implications for $21 \mathrm{st}$ century predictions by species distribution models. - Global Change Biol. 18: 1698-1713.

Viau, A. et al. 2006. Millennial-scale temperature variations in North America during the Holocene. - J. Geophys. Res. 111: $1-12$.

Webb, T. III 1993. Constructing the past from late-Quaternary pollen data: temporal resolution and a zoom lens space-time perspective. - In: Kidwell, S. M. and Behrensmeyer, A. K. (eds), Taphonomic approaches to time resolution in fossil assemblages. Paleontological Society, pp. 79-101.

Webb, T. III et al. 1981. Estimating plant abundances from pollen percentages: the use of regression analysis. - Rev. Palaeobot. Palynol. 34: 269-300.

Wiens, J. 1989. The ecology of bird communities. - Cambridge Univ. Press. 
Wilf, P. et al. 2001. Insect herbivory, plant defense, and early Cenozoic climate change. - Proc. Natl Acad. Sci. USA 98: 6221-6226.

Williams, J. W. et al. 2002. Rapid and widespread vegetation responses to past climate change in the North Atlantic region. - Geology 30: 971-974.

Williams, J. W. et al. 2004. Late-Quaternary vegetation dynamics in North America: scaling from taxa to biomes. - Ecol. Monogr. 74: 309-334.

Wisz, M. S. et al. 2013. The role of biotic interactions in shaping distributions and realised assemblages of species:

Supplementary material (Appendix ECOG-00779 at $<$ www. ecography.org/readers/appendix $>$ ). Appendix 1. implications for species distribution modelling. - Biol. Rev. 88: 15-30.

Yu, Z. 2007. Rapid response of forested vegetation to multiple climatic oscillations during the last deglaciation in the northeastern United States. - Quat. Res. 67: 297-303.

Zarnetske, P. L. et al. 2012. Biotic multipliers of climate change. - Science 336: 1516-1518.

Zhang, L. et al. 2011. Strong plant-soil associations in a heterogeneous subtropical broad-leaved forest. - Plant Soil 347: 211-220. 